\title{
Guidelines for training in percutaneous transluminal coronary angioplasty (PTCA)
}

\author{
Report of the Council of the British Cardiovascular Intervention Society (BCIS)
}

Coronary heart disease is responsible for considerable morbidity and is the single most common cause of death in the United Kingdom. Coronary artery bypass grafting (CABG) and percutaneous transluminal coronary angioplasty (PTCA) are of proven symptomatic benefit, and CABG improves prognosis in certain subgroups of patients. As preventive measures become more widely accepted the incidence of ischaemic heart disease may decrease but despite such a decrease the demand for revascularisation procedures (CABG or PTCA) will continue to rise for the foreseeable future. There are several reasons for this: the provision of cardiological services at district level is slowly improving, more patients are surviving myocardial infarction because of the use of thrombolytic agents, and there is increasing awareness of the benefits of revascularisation procedures. With advances in technology and operator experience the results of PTCA have improved, allowing more complex coronary artery disease to be treated in this way. Finally, as the number of patients who have had revascularisation procedures in the past increases, so the number potentially requiring re-investigation for recurrent symptoms will rise in the future.

Factors such as these will increase the number of referrals to centres providing revascularisation procedures and for this demand to be met an adequate number of PTCA operators must be trained. ${ }^{1}$ PTCA is a skilled procedure with success, failure, and complications relating to the operator's expertise and judgement and it is therefore essential that those wishing to undertake angioplasty are properly trained. This article provides guidelines aimed at optimising such training. As with other aspects of medicine it is the responsibility of all doctors performing PTCA, and particularly those training others in the technique, to ensure that in their institution the procedure can be performed safely and with a high likelihood of success.

\section{Angioplasty training}

In the United States the American Heart Association (AHA) and American College of Cardiology (ACC) have issued a joint report ${ }^{2}$ giving clear guidelines on the training required for an individual to perform angioplasty, the facilities a centre should have, and the workload a centre should undertake before a PTCA programme can be recognised. There are many differences in medical training and practice between the United Kingdom and the United States but the American guidelines provide a useful basis for our own recommendations.

\section{THE TRAINEE}

Before being accepted for PTCA training a doctor should have a sound basic training in general (internal) medicine, hold the MRCP, and have particular experience in managing patients with ischaemic heart disease. Guidelines for cardiological training in the United Kingdom ${ }^{3}$ and United States ${ }^{4}$ have been clearly defined. Prospective trainees must also be proficient in pacing procedures and cardiopulmonary resuscitation and have a thorough knowledge of the use of cardiovascular drugs. These requirements mean that angioplasty trainees will usually be junior cardiologists. Radiologists who intend to pursue careers in interventional catheterisation must have received training in, and be able to manage competently, the recognised cardiological complications of PTCA and it is the responsibility of those undertaking the training of such individuals to ensure that this minimum requirement is met.

Before being accepted for PTCA training all prospective trainees should be competent to perform right and left heart catheterisation and angiography and should have undertaken at least 300 unsupervised diagnostic coronary arteriograms. Before beginning training in PTCA the trainee must be able to perform and interpret coronary arteriograms safely and without assistance. All procedures undertaken should be recorded in a log-book and countersigned by a consultant experienced in catheterisation. Individuals should be accepted for angioplasty training by the consultant(s) who will subsequently take responsibility for training and who should ensure that the prospective trainee has fulfilled the general training requirements outlined above.

THE TRAINING

The training programme should ensure that the trainee becomes competent to assess the suitability of patients for PTCA. This assessment will inevitably vary to some extent between centres and will change with new developments in the technique. Rigid criteria for case selection are inappropriate but the recommendations of the ACC/AHA Task 
Force Report may be used as guidelines. ${ }^{2}$ The trainee must be familiar with the catheter laboratory and angioplasty equipment and understand the radiation implications of PTCA procedures and the means by which radiation exposure may be minimised. In addition, there should be a clear regimen for drug therapy before, during, and after angioplasty, however much this may be based on empirical grounds.

To a large extent the training programme is an apprenticeship and should aim to expose the trainee to as wide a variety of angioplasty experience as possible. Such an approach will ensure that the complications of angioplasty and their management become familiar to the operator.

In addition to performing PTCA effectively the trainee should be directly involved in the subsequent care of patients on the ward and after discharge from hospital, the assessment of an individual's outcome after the procedure, and the auditing of results for the centre as a whole. Trainees must keep abreast of the published reports on PTCA and should be allowed sufficient time to attend meetings. Each year a minimum of four days should be spent at meetings related to angioplasty in the United Kingdom or abroad.

Defining the minimum number of PTCA procedures that constitutes an acceptable training in angioplasty is difficult because individuals learn at different rates, case selection and available facilities will differ between centres, and the number of PTCA procedures performed annually in the United Kingdom means that some centres still have a relatively small workload. From figures supplied to the British Cardiovascular Intervention Society $21(49 \%)$ of the 43 NHS centres in the United Kingdom performed fewer than 150 angioplasties in $1990 .^{5}$ The recommendations of the AHA/ACC Task Force in the United States, ${ }^{2}$ and those of the Joint International Society and Federation of Cardiology/World Health Organisation Task Force, ${ }^{6}$ published in 1988, were that a minimum of 125 coronary angioplasty procedures are required, including 75 performed as a primary operator, before the individual is accredited as an independent operator. In a recent review of coronary angioplasty in the United Kingdom ${ }^{7}$ it was suggested that 50 procedures be performed before the trainee acts as a primary operator and that after this number has been attained an experienced angioplasty operator should be available nearby to advise and if necessary take over the procedure in case of difficulty. The number of these supervised procedures that are required before a trainee is regarded as a fully independent operator was not specified. The British Cardiovascular Intervention Society recommends that trainees in the United Kingdom be involved in 100 angioplasty procedures and be the primary operator on at least 50 of these, before they can be regarded as independent operators. All procedures and their outcome should be recorded in a log-book and certified by a recognised trainer.

\section{THE TRAINERS}

By now the experience gained by consultants who have trained themselves in angioplasty should be sufficient for all regional centres to have at least two consultants capable of training junior staff in angioplasty, thus ensuring continuity of services when one consultant is absent. The onus is on the trainer to provide adequate training and to ensure that this programme meets the requirements of both trainer and trainee. To maintain certification as a trainer in the United States requires an individual to perform at least 50 PTCA procedures per year as a principal operator. ${ }^{2}$ The British Cardiovascular Society makes the same recommendation for trainers in the United Kingdom.

\section{TRAINING CENTRES}

If one assumes that each centre has at least two principal operators and that each requires 50 PTCA cases per year to maintain experience, each training centre should undertake a minimum of 150 PTCA procedures each year if one trainee is to be trained as a primary operator. This allows 50 procedures to be undertaken by the trainee. At least 50 additional cases will be required if a second primary operator is to be trained. If trainers undertake some of their PTCA procedures in another institution the total required of the training centre may be slightly less.

The absence of on-site facilities for cardiac surgery is regarded as an absolute contraindication to PTCA in the United States ${ }^{2}$ but has been the subject of active debate in the United Kingdom. ${ }^{89}$ The British Cardiovascular Intervention Society regards on-site surgery as the strongly preferred option though it recognises that PTCA has been and can be performed satisfactorily without on-site surgery. However, units without surgery on-site must be able to demonstrate that there are formal arrangements for the prompt transfer of a patient to an operating theatre in an ambulance with staff and equipment that allow cardiac resuscitation and support. ${ }^{10} \mathrm{~A}$ centre without surgery on-site that satisfies these requirements and performs more than 150 PTCA procedures per year may be recognised for training. However, centres with on-site surgery may offer additional experience to the trainee. The presence of surgery on-site will ensure that the widest possible range of patients will be treated in the unit and thus the trainee will be exposed to angioplasty of a wide variety of lesions. It is important that a trainee has first hand experience of patients requiring emergency surgery during angioplasty so that he/she knows when to refer a patient and that he/she is involved in care right up to the time the patient enters the operating theatre. Also, easy availability of surgical colleagues for discussion about patients before angioplasty will help the trainee make informed and sensible decisions about the most suitable form of revascularisation procedure for individual patients. Whether cardiac surgery is on or off site there should be regular discussions between PTCA 
operators, especially those in training, and cardiac surgeons.

Any catheter laboratory undertaking angioplasty training should be equipped with a physiological measurement system, facilities for cardiopulmonary resuscitation, and a full range of angioplasty hardware. High quality $x$ ray imaging equipment capable of multiple views, including cranial and caudal angulations, should be available. Image freeze-frame and replay facilities must be instantly available. Because hard-copy images may need to be reviewed before the end of an angioplasty procedure rapid development of cineangiogram films should be possible. All centres undertaking angioplasty should provide good radiation protection for operators and catheter laboratory staff as well as patients. Centres should audit their angioplasty experiences so that results can be reviewed within their departments and accurate national data can be registered centrally with the United Kingdom Angioplasty Registry of the British Cardiovascular Intervention Society.

\section{Conclusion}

The British Cardiovascular Intervention Society makes the following recommendations for training in coronary angioplasty.

1 The trainee must have experience in general (internal) and cardiovascular medicine, and have performed and interpreted at least 300 unsupervised diagnostic coronary arteriograms before undertaking PTCA training.

2 The trainee should record diagnostic catheter procedures and PTCA procedures in a logbook.

3 The trainee must undertake at least 100 PTCA procedures, 50 of which must be as first operator, before being regarded as an independent operator.

4 Trainees should be trained in the selection of patients for PTCA and in their management after the procedure.

5 Training programmes should allow regular access to cardiac surgeons for discussion about the most appropriate form of revascularisation for individual patients.
6 A trainer should perform at least 50 PTCA procedures each year.

7 A training centre should undertake at least 100-150 PTCA procedures a year.

8 All centres undertaking PTCA should audit their procedures and are encouraged to contribute to the United Kingdom Angioplasty Registry held by the British Cardiovascular Intervention Society.

The recommendations made in this article are guidelines for establishing recognised training posts in coronary angioplasty in the United Kingdom. Since the competence of angioplasty operators, whether trainer or trainee, is to a large extent dependent on the number of procedures performed, the recommended numbers quoted should be regarded as an absolute minimum. The recommendations made are those of the Council of the British Cardiovascular Intervention Society and were discussed and accepted at the Edinburgh meeting of the Society in October 1989. They may require modification in future, in the light of further experience in the practice of coronary intervention procedures.

1 Training for coronary angioplasty. $\mathrm{Br} \mathrm{Med} J$ 1989;298: 768-9.

2 Guidelines for Percutaneous Transluminal Coronary Angioplasty. A report of the American College of Cardiology/American Heart Association Task Force on assessment of diagnostic and therapeutic cardiovascular procedures (Subcommittee on Percutaneous Transluminal Coronary Angioplasty). J Am Coll Cardiol 1988; 12:529-45.

3 Joint Committee on Higher Medical Training of the Royal College of Physicians. Recommendations for training in Cardiovascular Medicine. Royal College of Physicians. London, 1991.

4 Bethesda Conference on Adult Cardiology Training. J Am Coll Cardiol 1986;7:1205-6.

5 British Cardiovascular Intervention Society Report. Cardiac interventional procedures in the United Kingdom during interventional procedures in the
1990. Br Heart J 1992;68:434-6.

6 Report of the Joint International Society and Federation of Cardiology/World Health Organisation Task Force on Coronary Angioplasty. Eur Heart J 1988;9:1034-45.

7 Report of a working party of the British Cardiac Society. Coronary angioplasty in the United Kingdom. Br Heart $J$ 1991;66:325-31.

8 Parker DJ. Does angioplasty need on-site surgical cover? A surgeon's view. Br Heart J 1990;64:1-2.

9 Shaw TRD. Does angioplasty need on-site surgical cover? A physician's view. Br Heart J 1990;64:3-4.

10 Council of the British Cardiovascular Intervention Society. Surgical cover for percutaneous transluminal coronary angioplasty. $\mathrm{Br}$ Heart $J$ 1992;68:339-41. 